\title{
Put a (clear, goal-oriented, flexible and focused) contract on them! Some thoughts on selecting your pest contractors
}

\author{
Armando Mendez ${ }^{\ddagger}$, Suzanne Ryder ${ }^{\ddagger}$, Chris Swindells ${ }^{\S}$ \\ ‡ The Natural History Museum, London, United Kingdom \\ $\S$ Acheta Consulting Ltd., North Somerset, United Kingdom
}

Corresponding author: Armando Mendez (a.mendez@nhm.ac.uk)

Received: 16 May 2018 | Published: 04 Jul 2018

Citation: Mendez A, Ryder S, Swindells C (2018) Put a (clear, goal-oriented, flexible and focused) contract on them! Some thoughts on selecting your pest contractors. Biodiversity Information Science and Standards 2 : e26721. https://doi.org/10.3897/biss.2.26721

\section{Abstract}

In the last 20 years, IPM (Integrated Pest Management) has become an increasingly important discipline within the area of Preventive Conservation.

Incorporating an IPM program is a complex task even for a small sized institution, so when facing the challenge of starting a monitoring, recording, quarantine and identification scheme whilst ensuring that IPM protocols and best practices cascading from a comprehensive IPM Policy are followed throughout the institution, most IPM professionals tend to focus on the invertebrate pest problem while relaying entirely on external pest control companies to deal with the parallel issue of vertebrate pest.

This talk will present a case study to show an example of the well-known correlation between vertebrate and invertebrate pest activity and their complex relations, providing a few tips for those new to IPM on how to spot potential infestation sources, identifying points of entry and following the clues to find the origin of infestations, demonstrating how the detective work is an essential part of the role of an IPM professional. Following up on the case study the talk will lead on to discuss the importance of stablishing secure and longlasting relationships with key position holders in your institution in order to influence key 
contracts. The talk will provide advice on how to help your colleagues in Building/Estates Management Departments (those usually being in charge of managing pest control contracts), select the best candidate for an institution that have chosen the IPM strategy of prevention and investment on investigation over the traditional methods of pest control.

The talk will also discuss issues of reaction versus prevention, legalities, preventive strategies, raising awareness of IPM in your institution, the importance of education and outreach and many other areas of IPM you always wanted to know about but were afraid to ask...

\section{Keywords}

conservation, pest management, quarantine, building management

\section{Presenting author}

Armando Mendez, Conservation Centre, Natural History Museum, London, UK 